\title{
HYPERBOLIC BILLIARDS AND STATISTICAL PHYSICS
}

\author{
N. CHERNOV AND D. DOLGOPYAT
}

\begin{abstract}
Mathematical theory of billiards is a fascinating subject providing a fertile source of new problems as well as conjecture testing in dynamics, geometry, mathematical physics and spectral theory. This survey is devoted to planar hyperbolic billiards with emphasis on their applications in statistical physics, where they provide many physically interesting and mathematically tractable models.
\end{abstract}

\section{Introduction}

Let $\mathcal{D}$ be a bounded domain on a plane or a $2 \mathrm{D}$ torus with piecewise smooth boundary. A billiard system in $\mathcal{D}$ is generated by a single particle moving freely inside $\mathcal{D}$ with specular reflections off the boundary $\partial \mathcal{D}$. The phase space of a billiard is a $3 \mathrm{D}$ manifold $\Omega$; the corresponding flow $\Phi^{t}: \Omega \rightarrow \Omega$ preserves the Liouville measure $\mu$ (which is uniform on $\Omega$ ). The space of all collision points makes a $2 \mathrm{D}$ cross-section $\mathcal{M} \subset \Omega$, and the corresponding return map $\mathcal{F}: \mathcal{M} \rightarrow \mathcal{M}$ (called billiard map) preserves a natural smooth probability measure $m$.

The billiard is hyperbolic if the flow $\Phi^{t}$ and the map $\mathcal{F}$ have nonzero Lyapunov exponents. The first class of hyperbolic billiards was introduced [86] by Sinai in 1970; he proved that if the boundary of $\mathcal{D}$ is convex inward, then the billiard is hyperbolic, ergodic, mixing and $\mathrm{K}$ mixing. He called such models dispersing billiards, now they are called Sinai billiards. They are also proven to be Bernoulli [43]. A few years later Bunimovich discovered $[9,10]$ that billiards in some domains $\mathcal{D}$

2000 Mathematics Subject Classification. Primary 37D50, Secondary 34C29, $60 \mathrm{~F}$.

Key words and phrases. Hyperbolic billiards, mixing, limit theorems.

We thank our advisor Ya. G. Sinai for introducing us to this subject and constant encouragement during our work. We thank our numerous collaborators, especially P. Balint, L. A. Bunimovich, R. de la Llave, G. Eyink, J. Jebowitz, R. Markarian, D. Szasz, T. Varju, L.-S. Young, and H.-K. Zhang, for many useful discussions on the subject of this survey. NC was partially supported by NSF. DD was partially supported by NSF and IPST. 
whose boundary is convex outward are also hyperbolic, due to a special 'defocusing mechanism'; the most celebrated example of his billiards is a stadium. More general classes of planar hyperbolic billiards are described in $[95,96,63,41]$; we refer to $[48,26]$ for extensive surveys on hyperbolic billiards.

Billiards differ from classical smooth hyperbolic systems (Anosov and Axiom A flows and maps) in several respects. First of all, many hyperbolic billiards have non-uniform expansion and contraction rates (for example, if the moving particle is almost tangent to a convex outward arc of the boundary, then it will 'slide', and many reflections will occur in rapid succession during a short interval of time; a similar phenomenon occurs in a cusp on the boundary). Only dispersing billiards without cusps have uniform expansion and contraction rates.

Second, and most importantly, the billiard dynamics have singularities - phase points where both map $\mathcal{F}$ and flow $\Phi^{t}$ become discontinuous and have unbounded derivatives. Singularities come from two sources:

(a) Grazing collisions. In this case nearby trajectories can land on boundary components that lie far apart.

(b) Corners. In this case two nearby trajectories can hit different boundary pieces converging to a corner and get reflected at substantially different angles.

Moreover, billiards without horizon (where the length of the free path between collisions is unbounded) have infinitely many singularity curves in phase space.

Singularities in billiards lead to an unpleasant fragmentation of phase space. More precisely, any curve in unstable cones gets expanded (locally), but the singularities may cut its image into many pieces, some of them shorter than the original curve, which then will have to spend time on recovering. This makes billiards similar to non-uniformly hyperbolic systems such as quadratic maps or Henon attractors.

In $[97,98]$ Young has proposed two general methods for studying nonuniformly hyperbolic systems: tower method and coupling method.

The first one generalizes well-known Markov partitions ([85]). The latter divide phase space into rectangles ('building blocks') that have a direct product structure and being moved under the dynamics intersect one another in a proper (Markov) way. In the tower method only one rectangle is used and its images only need to intersect itself in the Markov way for some (not all) iterations. The tower construction is thus more flexible than that of Markov partitions, but the symbolic dynamics it provides is just as good as the one furnished by a Markov partition. 
The coupling method is designed to directly control the dependence between the past and the future. Since points with the same past history form unstable manifolds, one wants to show that the images of any two curves in unstable cones have asymptotically the same distribution ([84]). To this end one partitions those curves into small subsets and pairs subsets of the first curve with those of the second one so that the images of the paired (coupled) points remain close to each other at all times (i.e. lie on the same stable manifold).

Both methods proved to be very efficient and produced many sharp results, as we describe below. We observe here that the tower method allows us to use functional analytic tools, in particular the theory of transfer operators $[3,71]$, which provide very precise asymptotic expansions. However the transfer operator approach requires a suitably defined space of functions (observables), which is sometimes too restrictive and dependent on the model at hand. For this reason the results obtained by the tower approach are often less explicit and the dependence on parameters of the model is less transparent. The coupling approach, being more elementary if less sophisticated, gives more explicit bounds and makes it easier to work with several systems at a time.

Our survey is organized as follows. Section 2 describes statistical properties of dispersing billiards. Section 3 is devoted to systems with slow mixing rates. Section 4 deals with billiards in the presence of external forces and discusses transport coefficients and their dependence on parameters. Section 5 is devoted to interacting billiard particles, and Section 6 deals with infinite volume billiards.

We will denote by $\mathcal{N}\left(0, \sigma^{2}\right)$ a normal random variable (vector) with zero mean and variance (covariance matrix) $\sigma^{2}$, and by $\rho_{\sigma^{2}}$ its density function.

\section{Dispersing billiards}

Dispersing billiards make the oldest and most extensively studied class of all chaotic billiards. They, arguably, have the strongest statistical properties among all billiards. We need to suppose that all corners have positively measured angles (no cusps) to guarantee uniform expansion and contraction rates.

The main difficulty in the studies of billiards is to cope with the destructive effect of fragmentation caused by singularities (we note that fragmentation may badly affect even relatively simple expanding maps so that they would fail to have good statistical properties [93]). In 
billiards, to cope with pathological fragmentation one imposes the following 'non-degeneracy' condition: there exist $m \in \mathbb{N}, \delta>0$, and $\theta_{0}<1$ such that for any smooth unstable curve $W$ of length less than $\delta$

$$
\sum_{i} \lambda_{i, m} \leq \theta_{0}
$$

where the sum runs over all smooth components $W_{i, m} \subset \mathcal{F}^{m}(W)$ and $\lambda_{i, m}$ is the factor of contraction of $W_{i, m}$ under $\mathcal{F}^{-m}$. Roughly speaking (1) says that there no too-degenerate singularities such as multiple passages through the corners. (1) always holds if there are no corners, i.e. if $\partial \mathcal{D}$ is smooth, because for grazing collisions the expansion factor approaches infinity on one side of each singularity line, but in it is unknown if the condition (1) holds in dispersing billiards with corners, nor if it is really necessary for the results presented below.

Let $\mathbb{B}_{\alpha}^{d}$ be the space of bounded $\mathbb{R}^{d}$-valued functions which are uniformly $\alpha$-Hölder continuous on each component of $\mathcal{M}$ where the map $\mathcal{F}$ is smooth. We write $\mathbb{B}_{\alpha}$ for $\mathbb{B}_{\alpha}^{1}$. Let $\overline{\mathbb{B}}_{\alpha}^{d}=\left\{A \in \mathbb{B}_{\alpha}^{d}: m(A)=0\right\}$. For any function $A \in \overline{\mathbb{B}}_{\alpha}^{d}$ we denote by $\sigma^{2}(A)$ the $d \times d$ (diffusion) matrix with components

$$
\sigma_{i j}^{2}(A)=\sum_{n=-\infty}^{\infty} m\left(A_{i}\left(A_{j} \circ T^{n}\right)\right)
$$

(if this series converges). Denote $S_{n}(x)=\sum_{k=0}^{n-1} A\left(\mathcal{F}^{k} x\right)$.

Theorem 1. The following four results hold under the condition (1): (a) (EXPONENTIAL MIXING $[97,18,20]$ ) There is a constant $\theta<1$ such that for every $A, B \in \overline{\mathbb{B}}_{\alpha}$ there is $C>0$ such that for all $n \in \mathbb{Z}$

$$
\left|m\left(A\left(B \circ \mathcal{F}^{n}\right)\right)\right| \leq C \theta^{|n|},
$$

which, in particular, implies the convergence of the series (2);

(b) (Functional Central Limit Theorem $[11,12,20])$ For $A \in$ $\overline{\mathbb{B}}_{\alpha}^{d}$ define a continuous function $W_{n}(t)$ by letting $W_{n}(k / n)=S_{k} / \sqrt{n}$ and interpolating linearly in between. Then $W_{n}(t)$ weakly converges, as $n \rightarrow \infty$, to a Brownian motion (Wiener process) with covariance matrix $\sigma^{2}(A)$.

(c) (Almost Sure InVARIANCE PRINCIPle $[66,20])$ There exist $\lambda>0$ such that for any $A \in \overline{\mathbb{B}}_{\alpha}$ we can find (after possibly enlarging the phase space) a Brownian motion (Wiener process) $w(t)$ with variance $\sigma^{2}(A)$ such that for almost all $x$ there is $n_{0}$ such that for $n \geq n_{0}$

$$
\left|S_{n}-w(n)\right|<n^{\frac{1}{2}-\lambda}
$$


(d) (Local Limit Theorem [91]) Suppose $A \in \overline{\mathbb{B}}_{\alpha}^{d}$ takes values in a closed subgroup $V \subset \mathbb{R}^{d}$ of rank $r$ and that there is no $B \in L_{m}^{2}(\mathcal{M})$ such that $A+B-B \circ \mathcal{F}$ belongs to a proper closed subgroup of $V$. Then for any continuous function $G$ with compact support and for any sequence $\left\{k_{n}\right\}$ such that $k_{n} / \sqrt{n} \rightarrow z \in \mathbb{R}^{d}$

$$
n^{r / 2} m\left(G\left(S_{n}-k_{n}\right)\right) \rightarrow \rho_{\sigma^{2}(A)}(z) \int F d l
$$

where $l$ is the Haar measure on $V$.

Parts (a)-(c) of Theorem 1 can be proved by both tower method and coupling method $([97,18,20,66])$. The only known proof of part (d) uses the tower construction. It would be useful to derive the last part also by the coupling approach, since then it would be applicable to systems depending on parameters.

If $\mathcal{A}$ is a function on $\Omega$, then standard reduction methods [73, 67] allow us to extend parts (b) and (c) to $S_{t}(X)=\int_{0}^{t} \mathcal{A}\left(\Phi^{s} X\right) d s$. The corresponding covariance matrix $\tilde{\sigma}^{2}(\mathcal{A})$ can be computed as follows. Consider the function $A(x)=\int_{0}^{\tau(x)} \mathcal{A}\left(\Phi^{s} x\right) d s$ on $\mathcal{M}$, where $\tau(x)$ is the length of the free path. Then

$$
\tilde{\sigma}^{2}(\mathcal{A})=\sigma^{2}(A) / \bar{\tau}
$$

where $\bar{\tau}=\pi \operatorname{Area}(\mathcal{D}) /$ length $(\partial \mathcal{D})$ is the mean free path in the billiard system [16].

It would be also nice to extend the part (c) to multidimensional observables, as the almost sure invariance principle readily implies other limit laws - the law of iterated logarithm, integral tests, etc. [20].

Problem 1. Prove almost sure invariance principle for $\mathbb{R}^{d}$ valued observables.

The above results can be applied to the Lorentz gas in $\mathbb{R}^{2}$. Consider a particle moving on the plane between a periodic array of fixed convex disjoint obstacles (scatterers). The natural phase space of this system is the unit tangent bundle to the plane minus the scatterers, and the natural invariant measure is infinite ( $\sigma$-finite). But since the dynamics commute with the $\mathbb{Z}^{2}$ action we can factor the latter out and reduce the system to a dispersing billiard on the unit torus.

Let $S_{n}$ be the center of the scatterer the particle hits at the $n$th collision. Then $S_{n}-S_{n-1}$ factors to a function $H\left(\mathcal{F}^{n-1} x\right)$ on the collision space $\mathcal{M}$ of the toral billiard. To apply Theorem 1 we need to assume that this billiard has finite horizon (a uniformly bounded free path), since otherwise $H(x)$ is unbounded and has infinite second moment. (This is not a technical restriction, the following result actually 
fails without the horizon assumption, see Section 3.) Let $q(t)$ be the position of the moving particle at time $t$.

Theorem 2. The following five results hold for finite horizon Lorentz gases:

(a) $([11,12]) S_{n} / \sqrt{n}$ converges weakly to $\mathcal{N}\left(0, \sigma^{2}\right)$ where

$$
\sigma_{i j}^{2}=\sum_{n=-\infty}^{\infty} m\left(H_{i}\left(H_{j} \circ \mathcal{F}^{n}\right)\right) \text {. }
$$

(b) $([11,12]) q(t) / \sqrt{t}$ converges to $\mathcal{N}\left(0, \sigma^{2} / \bar{\tau}\right)$.

(c) $([91]) m\left(S_{n}=0\right) \sim 1 /(2 \pi \operatorname{det}(\sigma) n)$.

(d) $([30,78]) S_{n}$ is recurrent.

(e) The Lorentz gas is ergodic with respect to its $\sigma$-finite invariant measure.

Parts (c) and (d) are recent. Part (e) follows from part (d) and [79].

Parts (c) and (d) indicate that $S_{n}$ behaves like a random walk.

Problem 2. Extend the analogy between $S_{n}$ and random walks (for instance, investigate the statistics of returns).

Some results in this direction are obtained in [40]. Results for geodesic flows on negatively curved surfaces can be found in [1].

\section{Slow mixing and non-standard limit theorems}

Here we describe some hyperbolic billiards with non-uniform expansion and contraction rates. Such are billiards with convex outward boundary components, semidispersing billiards (where the boundary is convex inward, but at some points its curvature vanishes, i.e. the boundary 'flattens'), as well as dispersing billiards with cusps. All these billiards have one feature in common - there are arbitrarily long series of reflections without expansion or contraction, which compromise the hyperbolicity.

Such series of 'idle' reflections occur in certain well defined regions in phase space. If $\hat{\mathcal{M}} \subset \mathcal{M}$ is their complement, then the return map $\hat{\mathcal{F}}: \hat{\mathcal{M}} \rightarrow \hat{\mathcal{M}}$ will have uniform expansion and contraction rates, so Young's methods will apply. The distribution of return times to $\hat{\mathcal{M}}$ then determines the rates of mixing:

Theorem 3. (a) ([28]) If $\mathcal{D}$ is a Bunimovich stadium (a table with $C^{1}$ boundary consisting of two semicircles and two parallel line segments) and $A, B \in \overline{\mathbb{B}}_{\alpha}$, then

$$
\left|m\left(A\left(B \circ \mathcal{F}^{n}\right)\right)\right| \leq \text { const } \cdot(\ln |n|)^{2} /|n| .
$$


The same bound holds for modified stadia bounded by two circular arcs and two non-parallel line segments.

(b)([28]) If $\mathcal{D}$ is a Bunimovich billiard table bounded by several circular arcs that do not exceed semicircles an $A, B \in \overline{\mathbb{B}}_{\alpha}$, then

$$
\left|m\left(A\left(B \circ \mathcal{F}^{n}\right)\right)\right| \leq \text { const } \cdot(\ln |n|)^{3} /|n|^{2} .
$$

(c) ([29]) Let $\mathcal{D}$ be a dispersing billiard table except the curvature of $\partial \mathcal{D}$ vanishes at two points $P, Q \in \partial \mathcal{D}$ such that the segment $P Q$ is a periodic orbit of period two. More precisely let the boundary $\partial \mathcal{D}$ contain two curves $y= \pm\left(|x|^{\beta}+1\right)$, where $\beta>2$, so that $P=(0,1)$ and $Q=(0,-1)$. Then for $A, B \in \overline{\mathbb{B}}_{\alpha}$,

$$
\left|m\left(A\left(B \circ \mathcal{F}^{n}\right)\right)\right| \leq \mathrm{const} \cdot(\ln |n|)^{a+1} /|n|^{a} \text { where } a=\frac{\beta+2}{\beta-2} .
$$

The logarithmic factors here are an artifact of the method used; they can presumably be removed [22] by approximating the map $\mathcal{F}$ on $\mathcal{M} \backslash \hat{\mathcal{M}}$ with a Markov chain (the region $\mathcal{M} \backslash \hat{\mathcal{M}}$ consists of countably many 'cells' that make almost a Markov partition). The bound (5) is expected for dispersing billiards with cusps [61], but this case turns out to be much harder; it is currently under investigation [27].

If correlations decay like $\mathcal{O}(1 / n)$, as in Bunimovich stadia, the series (2) is likely to diverge, so the central limit theorem is likely to fail. This happens because the main contribution to the sum $S_{n}$ comes from long series of (highly correlated) reflections without expansion or contraction. Again, we can employ the return map $\hat{\mathcal{F}}: \hat{\mathcal{M}} \rightarrow \hat{\mathcal{M}}$ and replace the given observable $A$ with its 'cumulative' version

$$
\bar{A}(x)=\sum_{n=0}^{R(x)-1} A\left(\mathcal{F}^{n} x\right),
$$

where $\hat{\mathcal{F}}(x)=\mathcal{F}^{R(x)}(x)$, i.e. $R(x)$ is the first return time (to $\hat{\mathcal{M}}$ ), but such $\bar{A}$ will usually be unbounded and have heavy tails.

First studies of limit laws for observables with heavy tails were undertaken by Aaronson and Denker [2] for systems with Markov partitions. Their results were extended to non-uniformly hyperbolic maps with Young towers by Balint and Gouezel [4]; they gave an abstract criterion for convergence to a Gaussian law under a non-classical normalization (the case which is most relevant for billiards).

Balint and Gouezel [4] redefined $R(x)$ in (6) to be the first return time to the only rectangle in Young's tower and proved a limit theorem under the assumption that $\bar{A}$ has a distribution in a non-standard domain of attraction of Gaussian law. They applied this criterion to a Bunimovich 
stadium bounded by two semicircles of radius 1 and two line segments $\Gamma_{1}$ and $\Gamma_{2}$ of length $L>0$ each: given a Hölder continuous observable $A \in C^{\alpha}(\mathcal{M})$, denote by

$$
I(A)=\frac{1}{2 L} \int_{\Gamma_{1} \cup \Gamma_{2}} A(s, \mathbf{n}) d s
$$

its average value on the set of normal vectors $\mathbf{n}$ attached to $\Gamma_{1}$ and $\Gamma_{2}$. (A slower decay of correlations for the stadium, compared to other Bunimovich billiards, is caused by trajectories bouncing between two flat sides of $\mathcal{D}$ and $I(A)$ represents the contribution of such trajectories.)

Theorem 4. The following results hold for Bunimovich stadia:

(a) If $I(A) \neq 0$ then $S_{n} / \sqrt{n \ln n} \rightarrow \mathcal{N}\left(0, \sigma^{2}(A)\right)$, where

$$
\sigma^{2}(A)=\frac{4+3 \ln 3}{4-3 \ln 3} \times \frac{[I(A)]^{2} L^{2}}{4(\pi+L)} .
$$

(b) If $I(A)=0$, then there is $\sigma_{0}^{2}>0$ such that $S_{n} / \sqrt{n} \rightarrow \mathcal{N}\left(0, \sigma_{0}^{2}\right)$.

As before, the approach of [67] allows us to extend this result to flows.

The abstract criterion of [4] should be applicable to a large number of systems. One of them is a periodic Lorentz gas without horizon [92]. In this case orbits which never collide with the scatterers lie in a finite number of families of corridors $\Pi_{i} \subset \mathbb{R}^{2}$. The projection of each corridor onto the torus is a strip bounded by two periodic orbits (which in general case correspond to fixed points of the collision map $\mathcal{F})$. Let $w_{i}$ denote the vector joining the successive collisions along the bounding orbits for the corridor $\Pi_{i}$ (we call it a flight vector). Let also $f_{i}$ denote a vector parallel to $w_{i}$ but whose length equals the width of $\Pi_{i}$. Consider a nonnegative quadratic form

$$
Q(v)=\frac{1}{\operatorname{length}(\partial \mathcal{D})} \sum_{i}\left|w_{i}\right|\left\langle f_{i}, v\right\rangle^{2},
$$

it corresponds to a $2 \times 2$ symmetric positive semidefinite matrix $\sigma^{2}$.

Theorem 5 ([92]). Suppose there are at least two non-parallel corridors in a Lorentz gas without horizon. Then $\sigma^{2}>0$ and

(a) $S_{n} / \sqrt{n \ln n} \rightarrow \mathcal{N}\left(0, \sigma^{2}\right)$.

(b) If $k_{n} / \sqrt{n \ln n} \rightarrow z$ then $n \ln n \cdot m\left(S_{n}=k_{n}\right) \rightarrow \rho_{\sigma^{2}}(z)$.

(c) $S_{n}$ is recurrent.

(d) The Lorentz gas is ergodic with respect to its $\sigma$-finite invariant measure. 
Problem 3. Prove a functional central limit theorem in the setting of $[4]$.

Solving this problem would lead to a complete asymptotic description of the flight process in Lorentz gases without horizon.

\section{Transport coefficients}

Here we begin the discussion of billiard-related models of mathematical physics. The simplest one is a billiard $\mathcal{D}$ where the particle moves under an external force

$$
\dot{v}=F(q, v) .
$$

Such systems were investigated in [19] under the assumptions that $\mathcal{D}$ is the torus with a finite number of disjoint convex scatterers and finite horizon. To prevent unlimited acceleration or deceleration of the particle, it was assumed that there was an integral of motion ("energy") $\mathcal{E}(q, v)$ such that each ray $(q, \alpha v), \alpha \in \mathbb{R}_{+}$intersects each level surface $\{\mathcal{E}=c\}$ in exactly one point. To preserve hyperbolicity, it was assumed that $\|F\|_{C^{1}}$ is small.

Such forces include potential forces $(F=-\nabla U)$, magnetic forces $(F=B(q) \times v)$ and electrical forces with the so called Gaussian thermostat:

$$
F=E(q)-\frac{\langle E(q), v\rangle}{\|v\|^{2}} v .
$$

Fix an energy surface $\{\mathcal{E}(q, v)=$ const $\}$ containing a point with unit speed. Under our assumptions on $\mathcal{E}$ this level surface is is diffeomorphic to the unit tangent bundle $\Omega$ over $\mathcal{D}$ and the collision space $\mathcal{M}_{F}$ is diffeomorphic to $\mathcal{M}$. Denote by $\mathcal{F}_{F}: \mathcal{M}_{F} \rightarrow \mathcal{M}_{F}$ the corresponding return map.

Theorem 6 ([19]). $\mathcal{F}_{F}$ has a unique SRB (Sinai-Ruelle-Bowen) measure $m_{F}$, i.e. for Lebesgue almost every $x \in \mathcal{M}_{F}$ and all $A \in C\left(\mathcal{M}_{F}\right)$

$$
\frac{1}{n} \sum_{i=0}^{n-1} A\left(\mathcal{F}_{F}^{i} x\right) \rightarrow \int_{\mathcal{M}_{F}} A d m_{F} .
$$

The map $\mathcal{F}_{F}$ is exponentially mixing and satisfies the Central Limit Theorem (cf. Theorem 1).

As usual one can derive from this the existence (and uniqueness) of the SRB measure $\mu_{F}$ for the continuous time system.

Another interesting modification of billiard dynamics results from replacing the "hard core" collisions with the boundary by interaction 
with a "soft" potential near the boundary. We do not describe such systems here for the lack of space referring the reader to [60].

Theorem 6 implies the existence of various transport coefficients for planar Lorentz gas with finite horizon. For example, consider a thermostated electrical force (9) with a constant field $E(q)=E=$ const, and let $m_{E}$ denote the SRB measure on the $\{\mathcal{E}=1 / 2\}$ energy surface.

Theorem 7 ([24]). There is a bilinear form $\omega$ such that for $A \in$ $C^{\alpha}(\mathcal{M})$

$$
m_{E}(A)=m(A)+\omega(A, E)+o(\|E\|) .
$$

To illustrate these results, let $q_{n}$ denote the location of the particle on the plane at its $n$th collision, then Theorem 6 implies for almost all $x$ the average displacement $\left(q_{n}-q_{0}\right) / n$ converges to a limit, $J(E)$, i.e. the system exhibits an electrical current. Theorem 7 implies

$$
J(E)=M E+o(\|E\|) \quad(\text { Ohm's Law }) .
$$

where $M$ is a $2 \times 2$ matrix, see below.

One interesting open problem is to study the dependence of the measure $m_{F}$ of the force $F$, for example the smoothness of $m_{E}$ as a function of the electrical field $E$. For hyperbolic maps without singularities SRB measure depends smoothly on parameters $[51,76,77]$. For systems with singularities the results and methods of [24] demonstrate that the SRB measure is differentiable at points where it has smooth densities (e.g. $E=0$ in the previous example).

In fact there is an explicit expression for the derivative (Kawasaki formula). To state it let $\mathcal{F}_{\varepsilon}$ be a one-parameter family of maps such that $\mathcal{F}_{0}=\mathcal{F}$ has a smooth SRB measure and for small $\varepsilon$ the map $\mathcal{F}_{\varepsilon}$ has an SRB measure $m_{\varepsilon}$, too, and the convergence to the steady state $m_{\varepsilon}$, in the sense that if $\nu$ is a smooth probability measure on $\mathcal{M}$ and $A \in C^{\alpha}(\mathcal{M})$ then $\nu\left(A \circ \mathcal{F}_{\varepsilon}^{n}\right) \rightarrow m_{\varepsilon}(A)$, is exponential in $n$ and uniform in $\varepsilon$. Let $X=\left.\frac{d}{d \varepsilon}\right|_{\varepsilon=0}\left(\mathcal{F}_{\varepsilon} \circ \mathcal{F}^{-1}\right)$. Then

$$
\left.\frac{d}{d \varepsilon}\right|_{\varepsilon=0} m_{\varepsilon}(A)=-\sum_{n=0}^{\infty} \int_{\mathcal{M}} \operatorname{div}_{m}(X) A\left(\mathcal{F}^{n} x\right) d m(x) .
$$

For the constant electrical field $E$ the Kawasaki formula reads $\left.\frac{d}{d E}\right|_{E=0} J(E)=$ $\frac{1}{2} \sigma^{2}$, where $\sigma^{2}$ is defined by (4). Hence

$$
J=\frac{1}{2} \sigma^{2} E+o(\|E\|),
$$

which is known in physics as Einstein relation.

On the other hand numerical experiments [8] seem to indicate that $J(E)$ is not smooth for $E \neq 0$. Similar lack of smoothness is observed 
in $([44,45,47])$ for expanding interval maps, but the billiard case seems to be more complicated. Indeed the smoothness of SRB measures (or the lack thereof) seems to be intimately related to the dynamics of the singularity set. For 1D maps the singularity set is finite whereas for $2 \mathrm{D}$ maps the singularity set is one-dimensional, and so one can expect some statistics for the evolution of that set.

Problem 4. Prove that the SRB measure, as a function of parameters, is not smooth (generically). Derive relations between its Hölder exponent near a given parameter value and other dynamical invariants, such as Lyapunov exponents, entropy, etc.

A related issue is the dependence of infinite correlation sums, such as the one in (10), on the geometry of the billiard table. This issue was addressed in [23]. Given a domain $\mathcal{D} \subset \mathbb{T}^{2}$, an additional round scatterer is placed in $\mathcal{D}$ with a fixed radius $R>0$ and a (variable) center $Q$; then one gets a family of billiard maps $\mathcal{F}_{Q}$ acting on the same collision space $\mathcal{M}$ and having a common smooth invariant measure $m$. For any smooth functions $A, B$ on $\mathcal{M}$ let

$$
\sigma_{A, B}^{2}(Q)=\sum_{n=-\infty}^{\infty} m\left(A\left(B \circ \mathcal{F}_{Q}^{n}\right)\right)
$$

It is proven in $[23]$ that $\sigma_{A, B}^{2}(Q)$ is a log-Lipschitz continuous function of $Q$ :

$$
\left|\sigma_{A, B}^{2}\left(Q_{1}\right)-\sigma_{A, B}^{2}\left(Q_{2}\right)\right| \leq \text { const } \Delta \ln (1 / \Delta), \quad \text { where } \Delta=\left\|Q_{1}-Q_{2}\right\| .
$$

Problem 5. Is (13) an optimal bound?

Problem 6. Extend the analysis of [23] to dissipative systems studied in [19].

In particular is it true that the dependence on parameters is typically more regular for conservative systems?

Problem 7. Consider the class $\mathbb{S}$ of all Sinai billiard tables on $\mathbb{T}^{2}$ and deform a given table $\mathcal{D}$ continuously in $C^{4}$ so that it approaches the natural boundary of $\mathbb{S}$. Investigate the limit behavior of the diffusion matrix $\sigma^{2}(\mathcal{D})$.

If we only consider generic boundary points of $\mathbb{S}$, then this problem splits into three subproblems:

(a) What happens when two scatterers nearly touch each other?

(b) What happens when the boundary flattens so that a periodic trajectory with nearly zero curvature appears? 
(c) What happens when one of the scatterers shrinks to a point?

Analogues of Problem 7 were investigated for expanding maps [38] and for geodesic flows on negatively curved surfaces [13]. For Sinai billiards, only problem (c) has been tackled in [23], see Theorem 9(a) below. The first step towards problem (a) is to establish mixing bounds for billiards with cusps (for problem (b) this task has been accomplished in [29], see Theorem 3(c)).

One can also study the behavior of other dynamical invariants, such as entropy and Lyapunov exponents, see [16, 32, 48, 14].

\section{Interacting particles}

One may hope that after so many results have been obtained for one particle dynamics in dispersing billiards, a comparable analysis could be done for multi-particle systems, including models of statistical mechanics where the number of particles grows to infinity. However not much has been achieved up to now. Recently there has been a significant progress in the study of stochastically interacting particles $[52,94]$, but the problems involving deterministic systems appear to be much more difficult. One notable result is [70] where Euler equation is derived for Hamiltonian systems with a weak noise, however that particular noise is of a very special form, and its choice remains to be justified by microscopic considerations.

Regarding models with finitely many particles, the most celebrated one is a gas of hard balls in a box with periodic boundary conditions (i.e. on a torus $\mathbb{T}^{d}$ ). The ergodicity of this system is a classical hypothesis in statistical mechanics attributed to L. Boltzmann and first mathematically studied by Sinai [83, 86], see a survey [90]. The hyperbolicity and ergodicity for this system have been proven in fairly general cases only recently $[80,81]$, but a proof in full generality is not yet available.

Problem 8. Prove the ergodicity of $N$ hard balls on a torus $\mathbb{T}^{d}$ for every $N \geq 3$ and $d \geq 2$ and for arbitrary masses $m_{1}, \ldots, m_{N}$ of the balls.

The existing proofs $[80,81]$ cover 'generic' mass vectors $\left\{m_{1}, \ldots, m_{N}\right\}$ (apart from unspecified submanifolds of codimension one in $\mathbb{R}^{N}$ ). Besides, the existing proofs heavily rely on abstract algebraic-geometric considerations, and it would be important to find more explicit and dynamical arguments.

A system of $N$ hard balls on $\mathbb{T}^{d}$ can be reduced to semi-dispersing billiards in a $N d$-dimensional torus with a number of multidimensional 
cylinders removed. Now the considerations of Section 3 suggest that the rate of mixing for gases of hard balls is quite slow. Physicists estimated that correlation functions for the flow decay as $\mathcal{O}\left(t^{-d / 2}\right)$, see $[42,72]$.

Problem 9. Investigate mixing rate for gases of $N$ hard balls in $\mathbb{T}^{d}$ or $N$ hard disks on a Sinai billiard table.

An important feature of systems considered in statistical mechanics is that there are several different scales in space and time. This can complicate the study since the problem of interest tend to involve several 'levels' of parameters, but on the other hand one can expect certain simplifications; for example, Hamiltonian systems of $N$ particles which are not ergodic (and this is, generically, the case due to the KAM theory) may behave as ergodic in the thermodynamical limit $N \rightarrow \infty$ (see e.g. [31], Chapter 9). Another example is that some pathologies slowing the mixing rates can be suppressed on large time-space scales, thus the system may behave as strongly chaotic.

A significant progress in the study of multi-scale systems with chaotic fast motion has been achieved recently, see [39] and references wherein. In this section we describe the first rigorous result on multi-scale billiard systems [23].

Consider a system of two particles moving on a 2D torus with a finite number of fixed convex scatterers (we assume that the resulting region $\mathcal{D} \subset \mathbb{T}^{2}$ has finite horizon). Particles collide with the scatterers and with each other elastically. The first particle called $\mathbf{P}$ is a heavy disk of mass $M \gg 1$ and radius $R \sim 1$. The second particle called $\mathbf{p}$ is a dimensionless point of unit mass.

In equilibrium, the kinetic energies of $\mathbf{P}$ and $\mathbf{p}$ are comparable, and then $\mathbf{P}$ will move practically with constant velocity, without noticing p. A more interesting development occurs if the initial velocity of $\mathbf{P}$ is zero. Assume that the initial speed of $\mathbf{p}$ is 1 and that its initial state is chosen randomly from the unit tangent bundle over $\mathcal{D}$. Then the position $Q$ of $\mathbf{P}$ at time $t$ becomes a random process $Q_{M}(t)$. We want to describe the motion of $\mathbf{P}$ in the interior of $\mathcal{D}$ (before it has chance to reach $\partial \mathcal{D}$ ), so we fix a small $\delta>0$ and stop $\mathbf{P}$ once it comes within distance $\delta$ from $\partial \mathcal{D}$. Under a non-degeneracy condition on $\mathcal{D}$, see below, the following is proved:

Theorem 8 ([23]). As $M \rightarrow \infty$, the process $Q_{M}\left(\tau M^{2 / 3}\right)$ converges weakly to the solution of the following stochastic differential equation

$$
\ddot{Q}=\tilde{\sigma}(Q) \dot{w}
$$


where $\dot{w}$ is the white noise and the $2 \times 2$ matrix $\tilde{\sigma}(Q)$ is the positive square root of

$$
\tilde{\sigma}^{2}(Q)=\sigma^{2}(Q) / \bar{\tau}
$$

compare this to (3); here

$$
\bar{\tau}=\pi(\operatorname{Area}(\mathcal{D})-\operatorname{Area}(\mathbf{P})) /(\operatorname{length}(\partial \mathcal{D})+\operatorname{length}(\partial \mathbf{P}))
$$

is the mean free path for the fast particle $\mathbf{p}$ and

$$
\sigma^{2}(Q)=\sum_{n=-\infty}^{\infty} m\left(A\left(A \circ \mathcal{F}_{Q}^{n}\right)^{T}\right)
$$

where $\mathcal{F}_{Q}$ is defined before $E q$. (12) and $A \in \mathbb{B}^{2}$ is defined by (18) below.

The non-degeneracy condition mentioned above is $\sigma^{2}(Q)>0$ for all $Q$. This condition allows us to 'promote' the log-Lipschitz continuity of $\sigma^{2}$ given by (13) to the log-Lipschitz continuity of $\tilde{\sigma}$ and then show that the equation (14) is well posed. This illustrates the importance of Problems 5 and 6 for homogenization theory. The fact that $\sigma^{2}(Q)$ is non-degenerate, apart from a codimension infinity subset of $\mathbb{S}$, follows from [12].

To understand (14) observe that when $\mathbf{P}$ collides with $\mathbf{p}$ the tangential component of its velocity remains unchanged while the normal component changes as follows

$$
V_{\text {new }}^{\perp}=\frac{M-1}{M+1} V_{\text {old }}^{\perp}+\frac{2}{M+1} v_{\text {old }}^{\perp}=V_{\text {old }}^{\perp}+\frac{2}{M} v_{\text {old }}^{\perp}+\mathcal{O}\left(\frac{1}{M^{3 / 2}}\right)
$$

where $v_{\text {old }}^{\perp}$ is the normal component of the velocity of $\mathbf{p}$ (the estimate on the remainder term uses the fact that due to the energy conservation $M\|V\|^{2}+\|v\|^{2}=1$ the speed of $\mathbf{P}$ never exceeds $\left.1 / \sqrt{M}\right)$. Hence velocity of $\mathbf{P}$ after $n$ collisions equals

$$
V_{n}=\frac{2}{M} \sum_{i=1}^{n} v_{i}^{\perp}+\mathcal{O}\left(\frac{n}{M^{3 / 2}}\right)
$$

there $v_{i}^{\perp}$ is the normal component of the velocity of $\mathbf{p}$ before the $i$-th collision of $\mathbf{P}$ with $\mathbf{p}$. As we need to count all the collisions of $\mathbf{p}$, both with $\mathbf{P}$ and $\partial \mathcal{D}$, then (16) takes form

$$
V_{n}=\frac{2}{M} \sum_{i=1}^{n} A \circ \mathcal{F}^{i}+\mathcal{O}\left(\frac{n}{M^{3 / 2}}\right)
$$

where $\mathcal{F}$ is the collision map in our system of two particles and

$$
A=2 v^{\perp} \text { if } \mathbf{p} \text { collides with } \mathbf{P} \text { and } 0 \text { otherwise. }
$$


As $M \rightarrow \infty$, our system approaches the limit where $\mathbf{P}$ does not move $(Q \equiv$ const $)$ and $\mathbf{p}$ bounces off $\partial \mathcal{D} \cup \mathbf{P}$ elastically, thus its collision map is $\mathcal{F}_{Q}$. For this limiting system, Theorem $1(\mathrm{c})$ says that if $n=M^{\alpha} d \tau$, then

$$
\sum_{i=1}^{n} A \circ \mathcal{F}_{Q}^{i} \sim M^{\alpha / 2} \sigma(Q) d w(\tau)
$$

where $w(\tau)$ is the standard Brownian motion. Since $Q=\int V d t$ and the integral of the Brownian motion grows as $t^{3 / 2}$, it is natural to take $\alpha=2 / 3$ in (19), so that $M^{3 \alpha / 2} / M \sim 1$, cf. (16), and expect the limiting process to satisfy (14).

In the proof of Theorem 8 we had to show that the two-particle collision map $\mathcal{F}$ in (17) could be well approximated by the limiting billiard map $\mathcal{F}_{Q}$ in (19). While the trajectories of individual points under these two maps tend to diverge exponentially fast, the images of curves in unstable cones tend to stay close together, and we proved this by a probabilistic version of the shadowing lemma developed in [37]. Then we decomposed the initial smooth measure into one-dimensional measures on unstable curves (each curve $W$ with a smooth measure $\nu$ on it was called a standard pair) and adapted Young's coupling method to relate the image of each standard pair $(W, \nu)$ under the map $\mathcal{F}^{n}$ and that under $\mathcal{F}_{Q}^{n}$, as $n$ grows.

The system described above is a very simplified version of the classical Brownian motion where a macroscopic particle is submerged into a liquid consisting of many small molecules. In our model the liquid is represented by a single particle, but its chaotic scattering off the walls effectively replaced the chaotic motion of the molecules coming presumably from inter-particle interactions.

One feature of Theorem 8 which may be surprising at first glance is that the diffusion matrix $\sigma^{2}$ is position dependent - the feature one does not expect for the classical Brownian particle. The reason is that the size of $\mathbf{P}$ is comparable to the size of the container $\mathcal{D}$, so that typical time between successive collisions of $\mathbf{p}$ with $\mathbf{P}$ is of order one, hence $\mathbf{p}$ has memory of the previous collisions with $\mathbf{P}$ giving rise to a location dependent diffusion matrix. This dependence disappears if $\mathbf{P}$ is macroscopically small (but microscopically large!):

Theorem 9 ([23]). As $R \rightarrow 0$ we have

$$
\tilde{\sigma}^{2}(Q)=\frac{8 R}{3 \operatorname{Area}(\mathcal{D})} I+\mathcal{P}(Q) R^{2}+o\left(R^{2}\right)
$$


where $\mathcal{P}(Q)$ is a weighted Poincaré series. Furthermore, there is a function $M_{0}(R)$ such that if $M \rightarrow \infty$ and $R \rightarrow 0$ with $M>M_{0}(R)$, then $Q\left(\tau R^{-1 / 3} M^{2 / 3}\right)$ converges weakly to the process

$$
\sqrt{\frac{8}{3 \operatorname{Area}(\mathcal{D})}} \int_{0}^{\tau} w(s) d s
$$

where $w(s)$ is the standard Brownian Motion.

Observe that the formula (20) would easily follow if the collisions between $\mathbf{p}$ and $\mathbf{P}$ made a random Poisson process with intensity proportional to $2 R / \operatorname{Area}(\mathcal{D})$ (the inverse of the mean intercollison time).

We remark that since we have a single fast particle $\mathbf{p}$, its collisions with the boundary $\partial \mathcal{D}$ are the only source of chaos. If $\mathcal{D}$ is a convex smooth table, for example, then due to the presence of caustics [53] there is a positive probability that $\mathbf{p}$ and $\mathbf{P}$ will never meet, so Theorem 8 fails in that case.

Problem 10. Prove Theorems 8 and 9 for two particles in a square box.

In a square box, the fast particle may bounce off between two parallel sides for a long time without running into the disk, so the dynamics has slow mixing rates, cf. Section 3. According to the results of [4], see Theorem 4, one expects a non-standard normalization for most observables. However the observable given by (18) vanishes on $\partial \mathcal{D}$ (since the velocity of $\mathbf{P}$ does not change during the collisions of $\mathbf{p}$ with the walls), so we are actually in the context of Theorem 4(b), hence Central Limit Theorem may hold despite the overall slow mixing rates.

The extension of Theorem 9 to a square box leads (by using a standard reflection of the box across its boundary) to a new model - a fast particle moving on a plane with a periodic configuration of identical circular scatterers of radius $R \rightarrow 0$. This system is interesting in its own rights, but not much is known about its asymptotic properties as $R \rightarrow 0$. A lot of work has been done on the case where scatters are placed at random (see $[7,82]$ and references wherein) but the periodic case is much more complicated, see [46]. Even the distribution of the free path is a non-trivial task accomplished only recently [6].

The results of [23] extend, without much changes, to systems with several heavy disks and one fast particle, as long as the disks do not collide with each other or with the boundary of the table (of course this restricts the analysis to a fairly short interval of time). Let us, for example, formulate an analogue of Theorem 8 in this situation. Let $k$ be the number of heavy disks which are initially at rest. Then, 
after rescaling time by $\tau=M^{-2 / 3} t$, the velocity of the limiting process satisfies

$$
\frac{d}{d \tau}\left(\begin{array}{c}
V_{1} \\
\vdots \\
V_{k}
\end{array}\right)=\sigma_{Q_{1} \ldots Q_{k}} \dot{w(\tau)}
$$

where $\dot{w}$ is a standard $k$-dimensional white noise. Note that even though the heavy disks are not allowed to interact with each other directly, each one "feels" the presence of the others through the diffusion matrix $\sigma_{Q_{1} \ldots Q_{k}}$, which depends on the positions of all the disks.

A much more difficult problem arises if there are several fast particles.

Problem 11. Extend Theorems 8 and 9 to systems with several fast particles.

In this case the limiting $(M \rightarrow \infty)$ system consists of several noninteracting particles moving on the same dispersing billiard table (the heavy $\operatorname{disk}(\mathrm{s})$ will be "frozen" as $M=\infty$ ). Such a system can be reduced to a semidispersing billiard in a higher dimensional container, however that billiard will have very poor statistical properties. In fact, it will not be even fully hyperbolic - several of its Lyapunov exponents corresponding to the flow directions of the particles will vanish.

A more promising strategy for this case is to deal directly with the continuous time dynamics. Then the limiting system of several noninteracting fast particles is a direct product of one-particle billiard flows. To extend the results of [23] to this model we need to generalize their methods to the continuous time setting, and we also need good estimates for mixing rates of dispersing billiard flows.

Problem 12. Estimate the decay of correlations for dispersing billiard flows.

The studies of flow correlations are notoriously difficult (the main reason is that there is no expansion or contraction in the flow direction). Even for classical Anosov flows no estimates on correlations were available until the late 1990s. Only recently various estimates were obtained on the decay of correlations for smooth uniformly hyperbolic flows [17, 35, 59]. Some of them were just extended to nonuniformly hyperbolic flows [65], including Sinai billiards: it was shown [65] that for a 'prevalent' set of Sinai billiards with finite horizon, flow correlations decay faster than any polynomial function.

We expect that the flow correlations for Sinai billiards with finite horizon actually decay exponentially fast. Moreover, it appears that a sub-optimal ('stretched exponential') bound developed in [17] can be extended to billiard flows, and this is our work in progress. With some 
of these estimates, albeit less than optimal, we might be able to handle the above system of several fast particles.

Interestingly, the mixing rates of the billiard flow may not match those of the billiard map. For instance, in Sinai billiards without horizon the billiard map has fast (exponential) decay of correlations [18], but the flow is apparently very slowly mixing due to long flights without collisions [5]. On the contrary, in Sinai tables with cusps, the billiard map appears to have polynomial mixing rates, see Section 3, but the flow may very well be exponentially mixing, as the particle can only spend a limited time in a cusp. The same happens in Bunimovich billiards bounded only by circular arcs that do not exceed semicircles - the billiard map has slow mixing rates (Theorem 3), but the flow is possibly fast mixing, as sliding along arcs (which slows down the collision map) does not take much flow time.

The next step toward a more realistic model of Brownian motion would be to study several light particles of a positive radius $r>0$. (If there is only one light particle, such an extension is immediate since 'fattening' the light particle is equivalent to 'fattening' the disk $\mathbf{P}$ and the scatterers by the same width $r$.) It is however reasonable to assume that the light particles are much smaller than the heavy one, i.e. $r \ll R$. In this case one can presumably treat consecutive collisions as independent, so that in the limit $r \rightarrow 0$ the collision process becomes Markovian. An intermediate step in this project would be

Problem 13. Consider a system of $k$ identical particles of radius $r \ll 1$ moving on a dispersing billiard table $\mathcal{D}$. Let $E_{i}(t)$ denote the energy of the ith particle at time $t$. Prove that the vector

$$
\left\{E_{1}(\tau / r), E_{2}(\tau / r), \ldots E_{k}(\tau / r)\right\}
$$

converges, as $r \rightarrow 0$, to a Markov process with transition probability density given by the Boltzmann collision kernel [15]. This means that if particles $i$ and $j$ collide so that the angles between their velocities and the normal are in the intervals $\left[\phi_{i}, \phi_{i}+d \phi_{i}\right]$ and $\left[\phi_{j}, \phi_{j}+d \phi_{j}\right]$, respectively, with intensity

$$
\frac{\left|\sqrt{2 E_{i}} \cos \phi_{i}-\sqrt{2 E_{j}} \cos \phi_{j}\right| d \phi_{i} d \phi_{j}}{4 \pi^{2} \operatorname{Area}(\mathcal{D})},
$$

and then the particle $i$ transfers energy $E_{i} \cos ^{2} \phi_{i}-E_{j} \cos ^{2} \phi_{j}$ to the particle $j$.

The proof should proceed as follows. As long as the particles do not interact, the evolution of the system is a direct product of dynamics of individual particles. This holds true whenever the particle centers 
$q_{1}, \ldots, q_{k}$ are $>2 r$ units of length apart. Hence we need to investigate the statistics of visits of phase orbits to

$$
\Delta_{r}=\left\{\min _{i \neq j}\left\|q_{i}-q_{j}\right\| \leq 2 r\right\},
$$

which is a set of small measure. Visits of orbits of (weakly) hyperbolic systems to small measure sets have been studied in many papers, see $[36,50]$ and the references wherein We observe that Theorem 9 (a) is the first step in the direction of Problem 13.

Next, recall that in Theorem 8 we did not allow the disk $\mathbf{P}$ to come too close to the boundary $\partial \mathcal{D}$; this restricted our analysis to intervals of time $t=\mathcal{O}\left(M^{2 / 3}\right)$. During these times the speed of $\mathbf{P}$ remains small, $\|V\|=\mathcal{O}\left(M^{-2 / 3}\right)$, thus the system is still far from equilibrium, as $M\|V\|^{2}=\mathcal{O}\left(M^{-1 / 3}\right) \ll 1$.

Problem 14. Investigate the system of two particles $\mathbf{P}$ and $\mathbf{p}$ beyond the time of the first collision of $\mathbf{P}$ with $\partial \mathcal{D}$. In particular, how long does it take this system to approach equilibrium (where the energies of the particles become equal)?

There are two difficulties here. First, when $\mathbf{P}$ comes too close to the wall $\partial \mathcal{D}$, the mixing properties of the limiting $(M \rightarrow \infty)$ billiard system deteriorate, because a narrow channel forms between $\mathbf{P}$ and the wall. Once the fast particle $\mathbf{p}$ is trapped in that channel, it will bounce between $\mathbf{P}$ and the wall for quite a while before getting out; thus many highly correlated collisions between our particles occur, all pushing $\mathbf{P}$ in the same direction (off the wall). Thus we expect $\|\sigma(Q)\| \rightarrow \infty$ as the channel narrows. The precise rate of growth of $\|\sigma(Q)\|$ is important for the boundary conditions for equation (14), hence Problem 7 is relevant here.

The second difficulty is related to the accuracy of our approximations. The two particle system in Theorem 8 can be put in a fairly standard slow-fast format. Namely let $(q, v)$ denote the position and velocity of $\mathbf{p}$ and $(Q, V)$ those of $\mathbf{P}$. Put $\varepsilon=1 / \sqrt{M}$ and denote $x=(q, v /\|v\|)$ and $y=(Q, V)$ (note that $\|v\|$ can be recovered from $x$ and $y$ due to the energy conservation). Then $x$ and $y$ transform at the $n$th collision by

$$
\begin{aligned}
& x_{n+1}=T_{y_{n}}\left(x_{n}\right)+\mathcal{O}(\varepsilon) \\
& y_{n+1}=y_{n}+B\left(x_{n}, y_{n}\right)+\mathcal{O}\left(\varepsilon^{2}\right)
\end{aligned}
$$

If $T_{y}(x)$ is a smooth hyperbolic map, the following averaging theorem holds [39]. Let $W \ni\left(x_{0}, y_{0}\right)$ be a submanifold in the unstable cone, almost parallel to the $x$-coordinate space (i.e. $y \approx y_{0}$ on $W$ ), and 
such that $\operatorname{dim} W$ equals the dimension unstable subspace. Then for $|\ln \varepsilon| \ll n \ll 1 / \varepsilon$ and any smooth observable $A$ we have

$$
\int_{W} A\left(x_{n}, y_{n}\right) d x_{0}=\int A(x, y) d m^{y_{0}}(x)+\varepsilon \omega\left(A, y_{0}\right)+o(\varepsilon),
$$

where $m^{y_{0}}$ denotes the SRB measure of the map $T_{y_{0}}(x)$. This result is a local version of Theorem 7 (consider the case $y_{n} \equiv y_{0}$ !). In the presence of singularities, however, only a weaker estimate is obtained in [23]:

$$
\int_{W} A\left(x_{n}, y_{n}\right) d x_{0}-\int A(x, y) d m^{y_{0}}(x)=\mathcal{O}(\varepsilon|\ln \varepsilon|) .
$$

The extra factor $|\ln \varepsilon|$ appears because we have to wait $\mathcal{O}(|\ln \varepsilon|)$ iterates before the image of $W$ under the unperturbed (billiard) map becomes sufficiently uniformly distributed in the collision space, and at each iteration we have to throw away a subset of measure $\mathcal{O}(\varepsilon)$ in the vicinity of singularities where the shadowing is impossible. The weak estimate (23) was sufficient for time intervals $\mathcal{O}\left(M^{2 / 3}\right)$ considered in [23] since the corresponding error term in the expression for $V_{n}$, see $(17)$, is

$$
\mathcal{O}\left(\frac{n}{M} \times \frac{\ln M}{\sqrt{M}}\right)=\mathcal{O}\left(\frac{\ln M}{M^{5 / 6}}\right)
$$

because $n=\mathcal{O}\left(M^{2 / 3}\right)$. This error term is much smaller than the typical value of the velocity, $V_{n} \sim M^{-2 / 3}$.

However for $n \sim M$ the above estimate is not good as the error term would far exceeds the velocity itself. To improve the estimate (23) we have to incorporate the vicinity of singularities into our analysis. As the singularities are one-dimensional curves, we expect points falling into their vicinities to have a limit distribution, as $\varepsilon \rightarrow 0$, whose density is smooth on each singularity curve. Finding this distribution requires an accurate counting of billiard orbits passing near singularities. Such counting techniques have been applied to negatively curved manifolds [62], and we hope to extend them to billiards.

Another interesting model involving large mass ratio is so-called piston problem. In that model a container is divided into two compartments by a heavy insulating piston, and these compartments contain particles at different temperatures. If the piston were infinitely heavy, it would not move and the temperature in each compartment would remain constant. However, if the mass of the piston $M$ is finite the temperatures would change slowly due to the energy and momenta exchanges between the particles and the piston. There are several results about infinite particle case (see [21] and references wherein) but the case when the number of particles is finite but grows with $M$ is much 
more complicated (see [54]). On the other hand if the number of particles is fixed and $M$ tends to infinity then it was shown in $[88,69]$ under the assumption of ergodicity of billiard in each half of the container that after rescaling time by $1 / \sqrt{M}$ the motion of the piston converges to the Hamiltonian system

$$
\ddot{Q}=\Delta P:=\frac{K^{-} \ell}{2 \pi \operatorname{Area}\left(\mathcal{D}^{-}\right)}-\frac{K^{+} \ell}{2 \pi \operatorname{Area}\left(\mathcal{D}^{+}\right)}
$$

where $\mathcal{D}^{-}\left(\mathcal{D}^{+}\right)$is the part of the container to the left(right) of the piston $K^{-}\left(K^{+}\right)$is the energy of the particles in $\mathcal{D}^{-}\left(\mathcal{D}^{+}\right)$and $\ell$ is the length of the piston so that $\Delta P$ is the pressure difference. In particular if $\Delta P=0$ and piston is initially at rest then the system does not move significantly during the time $\sqrt{M}$ and the question is what happens on a longer time scale. For the infinite system it was shown in [21] that the motion converges to a diffusion process with the drift in the direction of the hotter gas. In the finite system (for example in a stadium container) this process will be accompanied by simultaneous heating of the piston so that the system may develop rapid $\left(\dot{Q} \sim \frac{1}{\sqrt{M}}\right)$ oscillations. A similar phenomenon was observed numerically in [25] for a system of $M^{2 / 3}$ particles in a $3 \mathrm{D}$ container. Those oscillations may be responsible for the fact that the system of [25] approaches its thermal equilibrium in $t \sim M^{a}$ units of time with some $1<a<2$ (computer experiments showed that $a \approx 1.7$ ).

In our model of two particles the formula (17) suggests that the time of relaxation to equilibrium is of order $M$, as in $n \sim M$ collisions the heavy disk will reach its maximum velocity $\left\|V_{n}\right\| \sim \sqrt{n} / M=1 / \sqrt{M}$; to prove this we need to improve our approximations along the above lines.

\section{Infinite measure systems}

Here we discuss several systems with infinite invariant measure, which can serve as tractable models of some non-equilibrium phenomena.

In ergodic theory, systems with infinite ( $\sigma$-finite) invariant measure are often regarded as exotic and attract little attention. However, hyperbolic and expanding maps with infinite invariant measure appear, more and more often, in various applications. Recently Lenci $[55,56]$ extended Pesin theory and Sinai's (fundamental) ergodic theorem to unbounded dispersing billiard tables (regions under the graph of a positive monotonically decreasing function $y=f(x)$ for $0 \leq x<\infty)$, 
where the collision map, and often the flow as well, have infinite invariant measures.

Another example that we already mentioned is the periodic Lorentz gas with a diffusive particle, but this one can be reduced, because of its symmetries, to a finite measure system by factoring out the $\mathbb{Z}^{2}$ action (Section 2). The simplest way to destroy the symmetry is to modify the location (or shape) of finitely many scatterers in $\mathbb{R}^{2}$. We call these finite modifications of periodic Lorentz gases.

Theorem 10. Consider a periodic Lorentz gas with finite horizon. Then

(a) ([57]) Its finite modifications are ergodic.

(b) ([40]) Its finite modifications satisfy Central Limit Theorem with the same covariance matrix as the original periodic gas does.

The proof of part (a) is surprisingly short. Every finite modification is recurrent, because if it was not, then the particle would not come back to the modified scatterers after some time, so it would move as if in a periodic domain, but every periodic Lorentz gas is recurrent (Theorem 2). Ergodicity then follows by [79].

The proof of (b) uses an analogy with a simple random walk (already observed in Section 2). Recall the proof of Central Limit Theorem for finite modifications of simple random walks [89]. Let $\xi_{n}$ be a simple random walk on $\mathbb{Z}^{2}$ whose transition probabilities are modified at one site (the origin). Define $\tilde{\xi}_{n}$ as follows: initially we set $\tilde{\xi}_{0}=\xi_{0}=0$, for every $n \geq 0$ we put

$$
\tilde{\xi}_{n+1}-\tilde{\xi}_{n}= \begin{cases}\xi_{n+1}-\xi_{n} & \text { if } \xi_{n} \neq 0 \\ X_{n} & \text { if } \xi_{n}=0\end{cases}
$$

where $X_{n}= \pm e_{i}, i=1,2$, is a random unit step independent of everything else. Then $\tilde{\xi}_{n}$ is a simple random walk and

$$
\left|\xi_{n}-\tilde{\xi}_{n}\right| \leq \operatorname{Card}\left\{k \leq n: \xi_{k}=0\right\} .
$$

Since the number of visit to the origin depends only on the behavior of the walk outside of the origin the RHS of $(24)$ is $\mathcal{O}(\ln n)$ (see e.g. [33]) so Central Limit Theorem for $\tilde{\xi}_{n}$ implies Central Limit Theorem for $\xi_{n}$.

Let $\tilde{\mathbb{B}}_{\alpha}$ denote the space of $\alpha$ Hölder continuous functions on the collision space of our periodic Lorentz gas with a finite modification, such that every $A \in \tilde{\mathbb{B}}_{\alpha}$ differs from a periodic function only on a compact set and the periodic part has zero mean. Then if $x_{n}=\left(q_{n}, v_{n}\right)$ denotes the position and velocity of the particle after the $n$th collision 
and $x_{0}$ has a smooth distribution $\nu$ with compact support, then for $A \in \tilde{\mathbb{B}}_{\alpha}$

$\left|\mathbb{E}\left(A\left(x_{n}\right)\right)\right| \leq c \nu\left(\exists k \in[n-c \ln n, n]: q_{k}\right.$ is on a modified scatterer $)+\mathcal{O}\left(n^{-100}\right)$, where $c>0$ is a constant. The proof of Theorem $1(\mathrm{~d})$ given in [91] allows us to estimate the first term here by $\mathcal{O}\left(\ln ^{\beta} n / n\right)$ for some $\beta>0$. The $\ln n$ factor is perhaps an artifact of the proof; on the other hand even for the much simpler case of a modified random walk one has $\mathbb{E}\left(A\left(\xi_{n}\right)\right) \sim c(A) / n$. This implies $\mathbb{E}\left(A\left(\xi_{0}\right) A\left(\xi_{n}\right)\right) \sim \bar{c}(A) / n$. Also there is a quadratic form $q(A)$ such that

$$
\mathbb{E}\left(A\left(\xi_{m}\right) A\left(\xi_{m+n}\right)\right) \sim \bar{q}(A) / n, \quad m, n \rightarrow \infty .
$$

Here we see a new feature of non-stationary systems which does not happen in finite ergodic theory. The correlation series $\sum_{n=1}^{\infty} \mathbb{E}\left(A\left(\xi_{m}\right) A\left(\xi_{m+n}\right)\right)$ diverges for all $m$ but Central Limit Theorem still holds, since the contribution of the off-diagonal terms to $\mathbb{E}\left(\xi_{n}^{2}\right)$ is much smaller than the contribution of near diagonal terms.

Finite modifications of periodic Lorentz gases are among the simplest billiards with infinite invariant measures, so we hope to move further in their analysis:

Problem 15. Extend (25) to finite modifications of periodic Lorentz gases (with finite horizon).

The reason for this simplicity is that finite modifications are restricted to a 'codimension two' subset of $\mathbb{R}^{2}$. The particle runs into modified scatterer very rarely, so that its limit distribution is the same as for the unperturbed periodic gas. The situation appears to be different for 'codimension one' modifications.

For example, consider a periodic Lorentz gas and make the particle move in the $N \times N$ box bouncing off its sides and off the scatterers in the box. Denote by $q_{N}(t)$ the position of the particle at time $t$.

Problem 16. Prove that $q_{N}\left(\tau N^{2}\right) / N$ converges, as $N \rightarrow \infty$, to the Brownian motion on the unit square with mirror reflections at its boundary.

If the box boundaries are symmetry axis of the Lorentz gas then the result follows easily from Theorem 1(b) but the general case appears more difficult. In fact if the boundaries of the box are not straight lines (so called rough boundaries) then one can expect the limit to be different due to trapping and it is an interesting problem to construct such counterexample.

As a more sophisticated example, consider a 'one-dimensional' Lorentz gas - a particle moving in an infinite strip $I=\{(x, y): 0 \leq y \leq 1\}$ 
(with identification $(x, 0)=(x, 1)$ ) and a periodic (in $x$ ) configuration of scatterers in $I$. Suppose a small external force $F$ acts by (8) in a compact domain $x_{\min } \leq x \leq x_{\max }$. Denote by $q_{F}(t)$ the position of the moving particle at time $t$.

Problem 17. Find the limit distribution of $q_{F}(\tau N) / \sqrt{N}$ as $N \rightarrow \infty$.

The analogy with the random walk [89] suggests that $q_{F}(\tau N) / \sqrt{N}$ should converge to $|\zeta| \eta$ where $\zeta$ and $\eta$ are independent, $\zeta$ is a onedimensional normal distribution $\mathcal{N}\left(0, \sigma^{2}\right)$ where $\sigma^{2}$ is the same as for the system without the field, and $\eta$ takes values \pm 1 , so that $\mathbb{P}(\eta=1) \sim$ $\mathbb{P}\left(q_{n}>0\right)$ depends on the evolution in the region $x_{\min } \leq x \leq x_{\max }$. One can further conjecture a functional limit theorem, namely that $q_{F}(\tau N) / \sqrt{N}$ converges to the so-called skew Brownian motion [49].

While the problems described above could be attacked along the lines of [40], the situation becomes much more difficult if modifications are less regular. In particular very little is known if the location of all scatterers is purely random (if there are infinitely many independent particles in a random Lorentz gas, ergodicity was proven by Sinai [87]).

Problem 18. Do results of Theorem 10 hold for random Lorentz gas?

The key question is the recurrence of the random Lorentz gas (this issue is irrelevant for infinite particle systems since if one particle wonders to infinity then another one comes to replace it, cf. [31], Chapter $9)$.

Lenci [58] uses Theorem 10 to show that recurrence holds for an 'open dense set' of Lorentz gases, but this remains to be shown for 'almost every' gas in any measure-theoretic sense.

Problem 17 brings us back to billiards with external forces, see Section 4. We assumed that (8) had an integral of motion. Without this assumption, the system would typically heat up (the particle accelerates indefinitely) or cool down (the particle slows down and stalls). It is interesting to determine which scenario occurs. Denote by $K(t)=\|v(t)\|^{2} / 2$ the particle's kinetic energy at time $t$.

Problem 19. Consider a Sinai billiards with a velocity-independent external force $\dot{v}=F(q)$. Is $\liminf _{t \rightarrow \infty} K(t)$ finite or infinite for most initial conditions?

The particular case of a constant force $F=$ const is long discussed in physics literature, see [74] and the references therein, but it is yet to be solved mathematically. This model is known in physics as Galton board - a titled plane with a periodic array of pins (scatterers) and a ball rolling on it under a constant (gravitational) force and bouncing 
off the pins. Due to the conservation of the total energy, the particle accelerates as it goes down the board. Physicists are interested in finding the limit distribution of its position (in a proper time-space scale). Heuristic arguments [68] indicate that $\|q(t)-q(0)\| \sim t^{2 / 3}$ and $\|v(t)\| \sim t^{1 / 3}$.

To address this problem observe that if we have a fast particle, i.e. $K(0)=\frac{1}{2 \varepsilon}$, then by rescaling the time variable by $s=t / \sqrt{\varepsilon}$ and denoting the rescaled velocity by $u=d x / d s$ we obtain a new equation of motion

$$
\frac{d u}{d s}=\varepsilon F(q)
$$

This system is of type (21) with fast variables $(q, u /\|u\|)$ and a slow variable $T=\|u\|^{2} / 2$. For random Lorentz gases heuristic arguments [74] suggests that in a new time variable $\tau=$ const $\cdot \varepsilon^{-2} s$ the limit evolution of $T$ will be given by

$$
\dot{T}=\frac{1}{2 \sqrt{2 T}}+(2 T)^{1 / 4} \dot{w}
$$

where $\dot{w}$ is a white noise. The same conclusion is reached in [34] for the geodesic flow on a negatively curved surface in the presence of a weak external force.

As a side remark, observe that the fast motion is obtained here by projecting the right hand side of (26) onto the energy surface, which gives us a thermostated force. In particular (11) plays an important role in the derivation of (27). This shows that the Gaussian thermostat (9), even though regarded as 'artificial' by some physicists, appears naturally in the analysis of weakly forced systems.

We return to the conjecture (27). In terms of our original variables, equation (27) says that $[K(t)]^{3 / 2}$ is the so called Bessel square process of index $4 / 3$, see $[75$, Chapter XI]. Since this process is recurrent, it is natural to further conjecture that there is a threshold $K_{0}>0$ such that for almost all initial conditions $\lim \inf _{t \rightarrow \infty} K(t) \leq K_{0}$. This conclusion apparently contradicts a common belief that the particle on the Galton board, see above, generally goes down and accelerates. Rather paradoxically, it will come back up (and hence slow down) infinitely many times! It appears that rigorous mathematics may disagree here with physical intuition, in a spectacular way.

The first step in solving this startling paradox would be to extend the averaging theorem (22) to billiards. 


\section{References}

[1] Aaronson J., Denker M., Distributional limits for hyperbolic infinite volume geodesic flows, Proc. Steklov Inst. 216 (1997) 174-185.

[2] Aaronson J., Denker M., Local limit theorems for partial sums of stationary sequences generated by Gibbs-Markov maps, Stoch. Dyn. 1 (2001), 193-237.

[3] Baladi V., Positive transfer operators and decay of correlations, Advanced Series in Nonlinear Dynamics, v. 16, World Scientific, River Edge, NJ, 2000.

[4] Balint P., Gouezel S., Limit theorems in the stadium billiard, preprint.

[5] Bleher, P. M., Statistical properties of two-dimensional periodic Lorentz gas with infinite horizon, J. Statist. Phys. 66 (1992), 315-373.

[6] Boca, F., Zaharescu, A., The distribution of the free path in the periodic two-dimensional Lorentz gas in the small-scatterer limit, manuscript.

[7] Boldrighini C., Bunimovich L. A., Sinai Ya. G. On the Boltzmann equation for the Lorentz gas, J. Stat. Phys. 32 (1983) 477-501.

[8] Bonetto F., Daems D., Lebowitz J., Properties of stationary nonequilibrium states in the thermostated periodic Lorentz gas I: the one particle system, $J$. Stat. Phys. 101 (2000), 35-60.

[9] Bunimovich L. A., Billiards that are close to scattering billiards, Mat. Sb. 94 (1974), 49-73.

[10] Bunimovich L. A., On the ergodic properties of nowhere dispersing billiards, Comm. Math. Phys. 65 (1979), 295-312.

[11] Bunimovich L. A., Sinai, Ya. G., Statistical properties of Lorentz gas with periodic configuration of scatterers, Comm. Math. Phys. 78 (1980/81), 479497.

[12] Bunimovich L. A., Sinai, Ya. G., Chernov, N. I., Statistical properties of twodimensional hyperbolic billiards, Russ. Math. Surveys 46 (1991), 47-106.

[13] Buser P. Geometry and spectra of compact Riemann surfaces, Progr. in Math. v. 106, Birkhauser, Boston, 1992.

[14] Burago D., Ferleger S., Kononenko A., Topological entropy of semi-dispersing billiards, Erg. Th. Dyn. Sys. 18 (1998), 791-805.

[15] Cercignani C., Illner R., Pulvirenti M., The mathematical theory of dilute gases, Appl. Math. Sciences, v. 106, Springer, New York, 1994.

[16] Chernov N., Entropy, Lyapunov exponents, and mean free path for billiards, J. Statist. Phys. 88 (1997), 1-29.

[17] Chernov N., Markov approximations and decay of correlations for Anosov flows, Ann. Math. 147 (1998), 269-324.

[18] Chernov N., Decay of correlations and dispersing billiards, J. Stat. Phys. 94 (1999), 513-556.

[19] Chernov N., Sinai billiards under small external forces, Ann. Henri Poincare 2 (2001), 197-236.

[20] Chernov N., Advanced statistical properties of dispersing billiards, preprint.

[21] Chernov N., On a slow drift of a massive piston in an ideal gas that remains at mechanical equilibrium, Math. Phys. Electron. J. 10 (2004), Paper 2, 18 pp.

[22] Chernov N., in preparation.

[23] Chernov N., Dolgopyat D., Brownian Brownian Motion - I, preprint. 
[24] Chernov, N., Eyink, G. L., Lebowitz, J. L., Sinai Ya. G., Steady-state electrical conduction in the periodic Lorentz gas, Comm. Math. Phys. 154 (1993), 569601.

[25] Chernov N., Lebowitz J., Dynamics of a massive piston in an ideal gas: Oscillatory motion and approach to equilibrium, J. Stat. Phys. 109 (2002), 507-527.

[26] Chernov N., Markarian R., Introduction to the ergodic theory of chaotic billiards, 2d ed. IMPA Math. Publ., 24th Brazilian Math. Colloquium, Rio de Janeiro, 2003.

[27] Chernov N., Markarian R., in preparation.

[28] Chernov N., Zhang H.-K., Billiards with polynomial mixing rates, Nonlinearity 18 (2005), 1527-1553.

[29] Chernov N., Zhang H.-K., A family of chaotic billiards with variable mixing rates, Stoch. Dyn. 5 (2005), 535-553.

[30] Conze, J.-P., Sur un critere de recurrence en dimension 2 pour les marches stationnaires, applications, Erg. Th. Dyn. Sys. 19 (1999), 1233-1245.

[31] Cornfeld I. P., Fomin S. V., Sinai Ya. G., Ergodic theory, Grundlehren der Mathematischen Wissenschaften 245 (1982) Springer, New York, 1982.

[32] Dahlqvist, P., The Lyapunov exponents in the Sinai billiards in the small scatterer limit, Nonlinearity 10 (1997), 159-173.

[33] Darling D. A., Kac M., On occupation times for Markoff processes, Trans. AMS 84 (1957), 444-458.

[34] de la Llave R., Dolgopyat D., Stochastic acceleration, in preparation.

[35] Dolgopyat D., On decay of correlations in Anosov flows, Ann. of Math. 147 (1998), 357-390.

[36] Dolgopyat D., Limit theorems for partially hyperbolic systems, Trans. AMS 356 (2004), 1637-1689.

[37] Dolgopyat D., On differentiability of SRB states for partially hyperbolic systems, Inv. Math. 155 (2004), 389-449.

[38] Dolgopyat D., Prelude to a kiss, Modern dynamical systems (ed. M. Brin, B. Hasselblatt and Ya. Pesin ), Cambridge Univ Press, 2004, 313-324.

[39] Dolgopyat D. Averaging and invariant measures, to appear in Moscow Math. $J$.

[40] Dolgopyat D., Szasz D., Varju T., Central Limit Theorem for locally perturbed Lorentz process, in preparation.

[41] Donnay V. J. Using integrability to produce chaos: billiards with positive entropy, Comm. Math. Phys. 141 (1991), 225-257.

[42] Erpenbeck J. J., Wood W. W., Molecular-dynamics calculations of the velocityautocorrelation function. Methods, hard-disk results, Phys. Rev. A 26 (1982), $1648-1675$.

[43] Gallavotti G., Ornstein D. S., Billiards and Bernoulli schemes, Comm. Math. Phys. 38 (1974), 83-101.

[44] Gaspard, P., Klages, R., Chaotic and fractal properties of deterministic diffusion-reaction processes, Chaos 8 (1998), 409-423.

[45] Gilbert, T., Ferguson, C. D., Dorfman, J. R., Field driven thermostated system: a non-linear multi baker map, Phys. Rev. E, 59 (1999), 364-371.

[46] Golse F., Wennberg, B., On the distribution of free path lengths for the periodic Lorentz gas-II, Math. Model. Numer. Anal., 34 (2000) 1151-1163. 
[47] Groeneveld, J., Klages, R., Negative and nonlinear response in an exactly solved dynamical model of particle transport, J. Stat. Phys. 109 (2002), 821861.

[48] Hard Ball Systems and the Lorentz Gas, Edited by D. Szasz. Encyclopaedia of Mathematical Sciences, v. 101 Springer, Berlin, 2000.

[49] Harrison J. M., Shepp L. A., On skew Brownian motion, Ann. Prob. 9 (1981), 309-313.

[50] Haydn N., Vaienti S., The limiting distribution and error terms for return times of dynamical systems, Discrete Cont. Dyn. Syst. 10 (2004), 589-616.

[51] Katok A., Knieper G., Pollicott M., Weiss H., Differentiability and analyticity of topological entropy for Anosov and geodesic flows, Inv. Math. 98 (1989), 581-597.

[52] Kipnis C. \& Landim C., Scaling limits of interacting particle systems, Grundlehren der Mathematischen Wissenschaften v. 320 Springer, Berlin, 1999.

[53] Lazutkin V. F., Existence of caustics for the billiard problem in a convex domain, Math. USSR (Izvestya) 37 (1973), 186-216.

[54] Lebowits J., Sinai Ya., Chernov N., Dynamics of a massive piston immersed in an ideal gas, Russian Math. Surv. 57 (2002) 1045-1125.

[55] Lenci, M., Semi-dispersing billiards with an infinite cusp-1, Comm. Math. Phys. 230 (2002), 133-180.

[56] Lenci, M., Semidispersing billiards with an infinite cusp-2,Chaos 13 (2003), $105-111$.

[57] Lenci M., Aperiodic Lorentz gas: recurrence and ergodicity, Erg. Th. Dyn. Sys. 23 (2003), 869-883.

[58] Lenci M., Typicality of recurrence for Lorentz gases, preprint.

[59] Liverani C., On contact Anosov flows, Ann. of Math. 159 (2004), 1275-1312.

[60] Liverani C., Interacting particles, in [48] pp. 179-216.

[61] Machta J., Power law decay of correlations in a billiard problem, J. Statist. Phys. 32 (1983), 555-564.

[62] Margulis G. A., On some aspects of the theory of Anosov systems, With a survey by Richard Sharp: Periodic orbits of hyperbolic flows, Springer Monographs in Math. Springer-Verlag, Berlin, 2004.

[63] Markarian R., Billiards with Pesin region of measure one, Comm. Math. Phys. 118 (1988), 87-97.

[64] Markarian R., Billiards with polynomial decay of correlations, Erg. Th. Dyn. Sys. 24 (2004), 177-197.

[65] Melbourne I., Rapid Decay of Correlations for Nonuniformly Hyperbolic Flows, to appear in Trans. AMS

[66] Melbourne I., Nicol M., Almost sure invariance principle for nonuniformly hyperbolic systems, Comm. Math. Phys. 260 (2005), 131-146.

[67] Melbourne I., Torok A., Statistical limit theorems for suspension flows, Israel J. Math. 144 (2004), 191-209.

[68] Moran, B., Hoover, W., Bestiale S. Diffusion in a periodic Lorentz gas. J. Stat. Phys. 48, (1987) 709-726.

[69] Neishtadt A. I., Sinai, Ya. G., Adiabatic piston as a dynamical system, J. Stat. Phys. 116 (2004) 815-820. 
[70] Olla S., Varadhan S. R. S., Yau H.-T., Hydrodynamical limit for a Hamiltonian system with weak noise, Comm. Math. Phys. 155 (1993), 523-560.

[71] Parry W., Pollicott M., Zeta functions and the periodic orbit structure of hyperbolic dynamics, Asterisque 187-188 (1990), 268 pp.

[72] Pomeau Y., Resibois P., Time dependent correlation function and mode-mode coupling theories, Phys. Rep. 19 (1975), 63-139.

[73] Ratner M., The central limit theorem for geodesic flows on $n$-dimensional manifolds of negative curvature, Israel J. Math. 16 (1973), 181-197.

[74] Ravishankar K., Triolo L., Diffusive limit of Lorentz model with uniform field from the Markov approximation, Markov Proc. Rel.Fields 5 (1999), 385-421.

[75] Revuz D. \& Yor M. Continuous martingales and Brownian motion, Grundlehren der Mathematischen Wissenschaften v. 293 Springer, Berlin, 1999.

[76] Ruelle D., Differentiation of SRB states, Comm. Math. Phys. 187 (1997), 227241.

[77] Ruelle D., Smooth dynamics and new theoretical ideas in nonequilibrium statistical mechanics, J. Stat. Phys. 95 (1999), 393-468.

[78] Schmidt K., On joint recurrence, CRAS Paris Ser. Math. 327 (1998), 837-842.

[79] Simanyi N., Towards a proof of recurrence for the Lorentz process, in Dynamical systems and ergodic theory (Warsaw 1986) (ed. K. Krzyzewski) Banach Center Publ., v .23, 265-276, PWN Warsaw, 1989.

[80] Simanyi N., Proof of the ergodic hypothesis for typical hard ball systems, Ann. Henri Poincaré 5 (2004), , 203-233.

[81] Simanyi N., Proof of the Boltzmann-Sinai ergodic hypothesis for typical hard disk systems, Invent. Math. 154 (2003), 123-178.

[82] Spohn H., Large scale dynamics of interacting particles, Springer, Berlin, 1991.

[83] Sinai Ya. G., On the foundations of the ergodic hypothesis for a dynamical system of statistical mechanics, Soviet Math. Dokl. 4 (1963), 1818-1822.

[84] Sinai Ya. G., Classical dynamic systems with countably-multiple Lebesgue spectrum-II, Izv. Akad. Nauk SSSR Ser. Mat. 30 (1966) 15-68.

[85] Sinai Ya. G., Markov partitions and U-diffeomorphisms. Funnk. Anal. Prilozh. 2 (1968) 64-89.

[86] Sinai Ya. G., Dynamical systems with elastic reflections: Ergodic properties of dispersing billiards, Russ. Math. Surv. 25 (1970), 137-189.

[87] Sinai, Ya. G., Ergodic properties of a Lorentz gas. Funkt. Anal. Prilozh. 13 (1979), 46-59.

[88] Sinai Ya. G., Dynamics of a massive particle surrounded by a finite number of light particles, Theor., Math. Phys. 121 (1999) 1351-1357.

[89] Szasz D., Telcs A., Random walk in an inhomogeneous medium with local impurities, J. Stat. Phys. 26 (1981), 527-537.

[90] Szasz D., Boltzmann's ergodic hypothesis, a conjecture for centuries? In: Hard ball systems and the Lorentz gas, 421-448, Encyclopaedia Math. Sci., Vol. 101, Springer, Berlin, 2000.

[91] Szasz D., Varju T., Local limit theorem for the Lorentz process and its recurrence in the plane, Erg. Th. Dyn. Sys. 24 (2004), 257-278.

[92] Szasz D., Varju T., Limit laws and recurrence for the planar Lorentz process with infinite horizon, preprint. 
[93] Tsujii M. Piecewise expanding maps on the plane with singular ergodic properties, Erg. Th. Dyn. Sys. 20 (2000), 1851-1857.

[94] Varadhan S. R. S. Entropy methods in hydrodynamic scaling, in Proceedings of ICM-94 196-208, Birkhauser, Basel, 1995.

[95] Wojtkowski M. Invariant families of cones and Lyapunov exponents, Erg. Th. Dyn. Sys. 5 (1985), 145-161.

[96] Wojtkowski M. Principles for the design of billiards with nonvanishing Lyapunov exponents, Comm. Math. Phys. 105 (1986), 391-414.

[97] Young L.-S. Statistical properties of dynamical systems with some hyperbolicity, Ann. Math. 147 (1998), 585-650.

[98] Young L.-S. Recurrence times and rates of mixing, Israel J. Math. 110 (1999), 153-188.

Nikolai Chernov, Department of Mathematics, University of AlABAma at Birmingham,, Birmingham AL 35294 USA

E-mail address: chernov@math.uab.edu

URL: http://www.math.uab.edu/chernov/

Dmitry Dolgopyat, Department of Mathematics, University of MaryLAND, COllege PARK MD 20742 USA

E-mail address: dmitry@math.umd.edu

$U R L:$ http://www.math.umd.edu/ dmitry/ 